\section{AB0122 RP53 INDUCES ECTODOMAIN SHEDDING OF TNF RECEPTOR 1 AND THEREBY INHIBITS INFLAMMATORY RESPONSES IN RHEUMATOID FIBROBLAST-LIKE SYNOVIOCYTES}

J. Kim ${ }^{1}$, H.S. Byun ${ }^{2}$, K. Kang ${ }^{2}$, S.-J. Yoo ${ }^{1}$, S.W. Kang ${ }^{1}$, G.M. Hur ${ }^{2}{ }^{1}{ }^{1}$ Internal Medicine; ${ }^{2}$ Pharmacology, Chungnam National University College of Medicine, Daejeon, Korea, Republic of Ireland

Background: Rheumatoid arthritis (RA) is a chronic inflammatory disease by autoimmune disorder that primarily affects joints. Usually RA has been treated with disease modifying anti-rheumatic drugs but biological response modifiers has developed the treatment of RA. Among these anti-tumour necrosis factor (TNF) agents were the first to be successfully used in treating RA. Various antiTNF- $\alpha$ therapy might be lead to substantial functional improvement in RA patients.

Objectives: We studied the isolated two polypeptides (Rp53, Rp54) from Rubia philippinensis, traditional medicine plant, about anti-inflammation effects in fibroblast-like synoviocytes (FLS) derived from patients with RA.

Methods: The effects of polypeptides on anti-inflammation were measured by cytokine assay kits (TNF- $\alpha$, IL-6). The underlying for NF-kB signalling pathway was examined by western blot and NF-kB reporter activity. We examined the effect of Rp53 on the formation of the TNFR1 signalling complex, recruitment of TRADD, RIP in response to TNF by immunoprecipitation experiments. To determine whether Rp53 induced TNF receptor 1 shedding were exposed to these compounds for 1 hour, and then culture media and cell lysates were analysed by Western blotting using anti-TNF receptor 1 antibody.

Results: Pretreatment with Rp53 resulted in a remarkable decrease of the secretion of TNF-induced proinflammatory cytokines TNF- $\alpha$ and IL-6 in RA-FLS. Rp53 strongly inhibited the nuclear factor $\mathrm{kB}(\mathrm{NF}-\mathrm{kB})$ signalling pathway induced by TNF- $\alpha$, but not that induced by IL- $1 \beta$. Analysis of the upstream signalling events affected by Rp53 revealed that it strikingly inhibited the TNF-induced recruitment of TNFR1-associated death domain protein (TRADD) and receptor-interacting protein (RIP) to TNFR1. Rp53 reduced the interaction with TNFR1 to TNF cytokines and enhanced the activation of the p38 mitogen-activated protein (MAP) kinase. Rp53, the polypeptides induced the proteolytic cleavage of TNF-R1 and its release into the culture medium by shedding of TNF receptor 1 ectodomain by TNF- $\alpha$-converting enzyme (TACE). Along with the TACE inhibitor TAPI-2, the p38 kinase inhibitor SB203580 suppressed the ectodomain shedding of TNF receptor 1 induced by Rp53.

Conclusions: Rp53 induces the TACE-dependent ectodomain shedding of TNF receptor 1 through the activation of p38 MAP kinase, and thereby inhibits the TNF- $\alpha$ induced NF- $\mathrm{KB}$ signalling pathway in RA-FLS cells.

Disclosure of Interest: None declared

DOI: 10.1136/annrheumdis-2018-eular.5178

\section{AB0123 EXPLORING THE METABOLOMIC PROFILE OF SYNOVIAL FLUID TO REFLECT THE DISEASE ACTIVITY IN RHEUMATOID ARTHRITIS}

J.K. Ahn ${ }^{1}$, J.-W. Hwang ${ }^{2}$, E.-M. Koh ${ }^{3}$, H.-S. Cha ${ }^{3}$, H. Jeong ${ }^{4}$, Y. Eun ${ }^{3}$, J. Lee ${ }^{3}$, H. Kim ${ }^{3} .{ }^{1}$ Kangbuk Samsung Hospital, Sungkyunkwan University School of Medicine; ${ }^{2}$ National Police Hospital; ${ }^{3}$ Samsung Medical Center, Sungkyunkwan University School of Medicine, Seoul; ${ }^{4}$ Soonchunghyang University School of Medicine, Bucheon, Gyeonggi-Do, Korea, Republic of Ireland

Background: Rheumatoid arthritis (RA) is a chronic, inflammatory disease that mainly affects the synovial joints. Metabolomics, which is defined as the comprehensive analysis of the small-molecule metabolites in a biological system, is a rapidly developing approach in biomarker research.

Objectives: This study was to determine whether there is variation in the synovial fluid (SF) metabolome in RA patients according to the degree of disease activity using gas chromatography/time-of-flight-mass spectrometry (GC/TOF-MS), in order to gain more insight into the pathologic metabolic alterations in RA.

Methods: SF samples were analysed in 47 patients with active RA, divided into moderately active- $(2.8<$ DAS28 ESR $<5.1, n=35$, male $28.6 \%$, mean age 55.1 years) and highly active group (DAS28-ESR $>5.1, n=12$, male $41.7 \%$, mean age 59.1 years). SF metabolite profiles of RA based on DAS28-ESR value were performed using GC/TOF MS, with univariate analysis such as student t-test and correlation analysis followed by multivariate analysis such as partial least-squares regression-discriminant analysis (PLS-DA) and pathway analyses such as metabolite set enrichment analysis.

Results: There was no differences in demographic data and medications use between two groups. A total of 123 metabolites were identified from samples. These 123 identified metabolites were classified into various chemical classes, such as amino acids ( $21 \%$ of identified metabolites), organic acids $(20 \%)$, sugar and sugar alcohols (19\%), fatty acids (15\%), amines (11\%), and phosphates (6\%). The SF metabolite profiles obtained from GC/TOF-MS analysis can distinguish moderately active group from highly active group. The variation values of the PLS-DA model are $R^{2} X$ of $0.171, R^{2} Y$ of 0.849 , and $Q^{2}$ of 0.504 , respectively, indicating strong explanation and prediction capabilities of the model. To find potential metabolic biomarkers reflecting the disease activity of RA, variable importance on projection (VIP) values of all the metabolites from the PLS-DA model were determined. Also, other statistical criteria including correlation coefficient and $P$ value were assessed. Twelve potential metabolites reflecting the disease activity of RA are found (2-hydroxyvalerate, fucose, tryptophan, indole-3 lactate, isothreonate, thymine, phenylalanine, lactose, arabitol, and mannose-6phosphate, citrate, oxoproline). In pathway analysis, we found six unique pathways, namely, fructose and mannose degradation, phenylalanine and tyrosine metabolism, citric acid cycle, galactose metabolism, tryptophan metabolism and pyrimidine metabolism that were significantly associated with disease activity in RA.

Conclusions: Synovial metabolite profiles are robustly altered along the disease activity of RA. Metabolomic approaches based on GC/TOF-MS could provide valuable information on the underlying pathogenic mechanisms of RA activity, and a novel perspective on the search of new biomarkers and drug targets.

Disclosure of Interest: None declared

DOI: 10.1136/annrheumdis-2018-eular.3286

\section{AB0124 DETECTION OF PRECURSORS OF RANK- OSTEOCLAST-LIKE CELLS (OLCS) IN PERIPHERAL BLOOD AND OLCS IN BONE TISSUE FROM RHEUMATOID ARTHRITIS PATIENTS}

K. Yokota ${ }^{1}$, S. Tanaka ${ }^{2}$, M. Sekikawa ${ }^{2}$, Y. Aizaki ${ }^{1}$, K. Sato ${ }^{1}$, H. Oda ${ }^{2}$, T. Mimura ${ }^{1}$ ${ }^{1}$ Department of Rheumatology and Applied Immunology, Faculty of Medicine; ${ }^{2}$ Department of Orthopaedic Surgery, Saitama Medical University, Iruma, Japan

Background: Previously, we reported that novel osteoclast-like cells (OLCs) were induced, both in vitro and in vivo, from mouse bone marrow-derived macrophages (BMMs) by addition of a combination of TNF $\alpha$ and IL- ${ }^{1}$. Recently, O'Brien et al showed that TNF/IL-6 can drive osteoclastogenesis in BMMs from RANKdeficient mice ${ }^{2}$

Objectives: We aimed to examine the differentiation of OLCs, which were induced by a combination of TNF $\alpha$ and IL- 6 from human peripheral blood mononuclear cells (PBMCs) and CD14 +monocytes and to identify differences in molecular expression patterns between OLCs and conventional osteoclasts. Furthermore, we identified OLCs and osteoclasts on the bone tissue of the joint in patients with rheumatoid arthritis (RA).

Methods: PBMCs and CD14 +monocytes from healthy volunteers and/or RA patients were stimulated with TNF $\alpha$ and IL- 6 or RANKL. Quantitative RT-PCR was used to measure mRNA expression levels of RANK, cathepsin $K$, calcitonin receptor, and dendritic cell-specific transmembrane protein. Prepared undecalcified tibial bone from 6 RA or osteoarthritis $(\mathrm{OA})$ patients undergoing joint surgery were stained by tartrate-resistant acid phosphatase (TRAP) staining and immunohistochemistry with anti-RANK antibody, expression of which were analysed. Osteoclasts and OLCs were identified as multinucleated TRAP+/RANK +cells and TRAP+/RANK- cells, respectively, adherent to the bone surface.

Results: The number of OLCs treated with a combination of TNF $\alpha$ and IL- 6 from PBMCs or CD14 +monocytes in RA patients was significantly increased compared to that in healthy volunteers. Expression levels of RANK mRNA was clearly up-regulated in osteoclasts, and was obviously down-regulated in OLCs compared to that in osteoclast precursors. In cancellous bone, the number of TRAP+/ RANK +osteoclasts was significantly increased in RA patients compared to that in OA patients. Interestingly, numerous TRAP+/RANK- OLCs were present in the cancellous bone of RA patients, while almost none were observed in the cancellous bone of OA patients.

Conclusions: The combination of TNF $\alpha$ and IL- 6 strongly induced the differentiation of OLCs from PBMCs or CD14 +monocytes in RA patients. OLCs was charac terised with TRAP+/RANK- multinucleated cells, which can distinguish conventional TRAP+/RANK +multinucleated osteoclasts. TRAP+/RANK- OLCs also were present in the bone tissue of RA patients. These results suggest that conventional osteoclasts and novel OLCs could be involved in the pathogenic mechanisms of inflammatory bone destruction such as RA.

\section{REFERENCES :}

[1] Yokota K, Sato K, Miyazaki T, Kitaura H, Kayama H, Miyoshi F, Araki $Y$, Akiyama $\mathrm{Y}$, Takeda $\mathrm{K}$, Mimura T. Combination of tumor necrosis factor $\alpha$ and interleukin- 6 induces mouse osteoclast-like cells with bone resorption activity both in vitro and in vivo. Arthritis Rheumatol 2014 Jan;66(1):121-9. 
[2] O'Brien W, Fissel BM, Maeda Y, Yan J, Ge X, Gravallese EM, Aliprantis AO, Charles JF. RANK-Independent Osteoclast Formation and Bone Erosion in Inflammatory Arthritis. Arthritis Rheumatol. 2016 Dec;68(12):28892900.

Disclosure of Interest: None declared

DOI: 10.1136/annrheumdis-2018-eular.5383

\section{AB0125 ANTI-DENGUE IGG ANTIBODY POSITIVITY AND RISK OF DEVELOPING RHEUMATOID ARTHRITIS: EVIDENCE FROM THE MALAYSIAN EPIDEMIOLOGICAL INVESTIGATION OF RHEUMATOID ARTHRITIS (MYEIRA) CASE-CONTROL STUDY}

L.K. $\operatorname{Tan}^{1,2}$, N.A. Ahmad Fauzi ${ }^{1}$, W. Sulaiman' ${ }^{2}$, O. Ali ${ }^{2}$, I.S. Lau ${ }^{3}$, H. Hussein ${ }^{4}$, N. S. Sharil ${ }^{4}$, S.C. Gun ${ }^{5}$, E. Mageswaren ${ }^{4}$, M.-S. Mohd-Said ${ }^{6}$, A. Mohd-Mokhtar ${ }^{7}$, A. Rosman ${ }^{3}$, M. Othman ${ }^{8}$, C. Bengtsson ${ }^{9}$, L. Alfredsson ${ }^{9}$, L. Klareskog ${ }^{10}$, S. Murad ${ }^{11}$, L. Padyukov ${ }^{10}$, C.L. Too ${ }^{1,10}{ }^{1}$ Allergy and Immunology Research Center, Institute for Medical Research, Ministry of Health Malaysia, Kuala Lumpur, ${ }^{2}$ Faculty of Medicine, University Kuala Lumpur Royal College of Medicine Perak, Ipoh, Perak; ${ }^{3}$ Department of Medicine, Selayang Hospital, Selangor, ${ }^{4}$ Department of Medicine, Putrajaya Hospital, Putrajaya; ${ }^{5}$ Department of Medicine, Hospital Tunku Ja'afar, Seremban, Negeri Sembilan; ${ }^{6}$ Faculty of Medicine, Universiti Kebangsaan Malaysia Medical Center, Kuala Lumpur, ${ }^{7}$ Department of Medicine, Tengku Ampuan Afzan Hospital, Kuantan, Pahang; ${ }^{8}$ Department of Medicine, Hospital Raja Perempuan Bainun, Ipoh, Perak, Malaysia; ${ }^{9}$ Institute of Environmental Medicine, Karolinska Institutet, ${ }^{10}$ Department of Medicine, Rheumatology Unit, Karolinska Institutet and Karolinska University Hospital, Stockholm, Sweden; ${ }^{11}$ Ministry of Health, Level 12th, Block E7, Parcel E, Federal Government Adminstrative Center, Putrajaya, Malaysia

Background: Arthralgia is one of the common symptoms seen in RA and in mosquito-borne viral diseases (dengue and chikungunya infections). Studies have reported that both dengue and chikungunya infections are associated with longterm persistent rheumatic symptoms including joints, muscle and bone pain.

Objectives: We investigated the association between anti-dengue $\operatorname{lgG}$ antibody positivity and risk of developing anti-citrullinated peptide antibody (ACPA)-positive and ACPA-negative RA in the multi-ethnic Malaysian population.

Methods: A total of 1147 early RA cases (515 Malay, 254 Chinese and 378 Indians) and 1519 age, sex and residential area matched population-based controls (1,023 Malay, 208 Chinese, and 288 Indians) were included in this study. Antidengue IgG antibody was determined by ELISA method. The presence of antidengue IgG antibody and risk of developing ACPA-positive/ACPA-negative RA were estimated by calculating the odds ratio (OR) with $95 \%$ confidence interval $(95 \% \mathrm{Cl})$.

Results: Our data demonstrated that $79.1 \%(n=1,003)$ and $77.1 \%(n=1,255)$ RA and control subjects were positive for anti-dengue $\lg G$ antibody, respectively. Data analysis revealed that the Chinese RA patients has highest frequency of anti-dengue IgG antibody (86.6\%) followed by the Indian (80.4\%) and Malay (74.4\%) RA patients while $83.7 \%, 87.5 \%$ and $73 \%$ Chinese, Indian and Malay healthy controls were positive for this antibody, respectively. The anti-dengue IgG antibody positivity was significantly associated with decreased risk of RA in the Indian population (OR $0.59,95 \% \mathrm{Cl}: 0.38-0.91, \mathrm{p}=0.02$ ) and particularly for the ACPA-positive subset of RA (OR $0.60,95 \% \mathrm{Cl} 0.37-0.96, \mathrm{p}=0.03$ ). Interestingly, we observed a non-significant increased risk for ACPA-positive RA in the Chinese (OR $1.49,95 \% \mathrm{Cl} 0.81-2.72$ ) and Malay populations (OR 1.06, 95\% Cl: $0.79-$ 1.41) with anti-dengue $\operatorname{lgG}$ antibody. No association was observed between ACPA-negative RA and the antibody positivity.

Conclusions: Our study describes the association between anti-dengue $\lg G$ antibody occurrence and ACPA-positive RA, but not ACPA-negative RA in an ethnicity-dependent manner. Future research is needed to explore the biological mechanisms behind these findings.

\section{REFERENCES:}

[1] Mohd Zim MA, et al. Chikungunya infection in Malaysia: Comparison with dengue infection in adults and predictors of persistent arthralgia. Journal of Clinical Virology 2013;56(2013):141-145.

[2] Gissel Garci' et al. Long-term persistence of clinical symptoms in dengueinfected persons and its association with immunological disorders. International Journal of Infectious Diseases 2011:15(2011):e38-e43.

Disclosure of Interest: None declared

DOI: 10.1136/annrheumdis-2018-eular.6190

\section{$\mathrm{AB} 0126$ \\ LOW PREVALENCE OF ANTIBODIES AGAINST MALONDIALDEHYDE-ACETALDEHYDE ADDUCTS IN SPANISH PATIENTS WITH RHEUMATOID ARTHRITIS}

L. Rodriguez-Martinez, E. Perez-Pampin, A. Mera-Varela, C. Conde, A. Gonzalez. Experimental and Observational Rheumatology, Instituto Investigacion SanitariaH. Clinico Universitario de Santiago, Santiago de Compostela, Spain

Background: Patients with rheumatoid arthritis (RA) present increased oxidative stress that leads to lipid peroxidation and the formation of malondialdehyde (MDA) and acetaldehyde (AA). These two compounds under oxidative stress form malondialdehyde-acetaldehyde (MAA) adducts with proteins, which are highly immunogenic. Recently, Thiele et al. ${ }^{1}$ described the presence of antibodies against human albumin MAA adducts in patients with established RA from the Veterans Affairs Rheumatoid Arthritis (VARA) registry. Of particular relevance was the reported presence of IgG anti-MAA antibodies in $92 \%$ of the patients, including $88 \%$ of the anti-CCP negative patients. These results suggest MAA adducts could contribute to the pathogenesis of RA and the anti-MAA antibodies could drastically reduce the number of patients with seronegative RA.

Objectives: To replicate the association of anti-MAA antibodies with RA and explore their value as biomarkers.

Methods: Sera from 515 Spanish patients with established RA that fulfilled the 1987 ACR classification criteria and from 274 healthy controls were included. Available information included history of smoking, anti-CCP status, and genotype of HLA-DRB1 and PTPN22 rs2476601. Human serum albumin MAA adducts and hexyl-MAA standard were chemically synthesised. Anti-MAA antibodies against the albumin MAA adducts were determined by indirect ELISA using isotype-specific secondary antibodies for $\lg G$, $\lg M$ and $\lg A$.

Results: Anti-MAA antibodies were detected in a small fraction of the RA patients, who had slightly increased antibody titers compared to healthy controls, $6.4 \%$ were positive for $\lg$ G, $15.7 \%$ for $\lg M$ and $8.0 \%$ for $\lg A$. The low prevalence of anti-MAA antibodies persisted in spite of multiple variations in the ELISA protocols including the use of different albumin sources, albumin MAA adducts produced in two different laboratories, and various secondary antibodies. IgM antiMAA antibody titers were increased in smokers compared to non-smokers. Moreover, the presence of $\lg M$ and of $\operatorname{IgA}$ anti-MAA antibodies were associated with anti-CCP and RF positivity.

Conclusions: Anti-MAA antibodies were detected in a small fraction of the Spanish RA patients, but their low sensitivity questions the value of these antibodies as biomarkers of RA. Due to the contradictory findings, additional studies should be performed that will need to address also the role of MAA adducts on RA pathogenesis.

\section{REFERENCE:}

[1] Thiele GM, et al. Arthritis Rheumatol 2015;67(3):645-55.

Acknowledgements: Supported by grants PI14/01651 and RD16/0012/0014 of the Instituto de Salud Carlos III (Spain) that are partially financed by the ERDF Disclosure of Interest: None declared

DOI: 10.1136/annrheumdis-2018-eular.4583

\section{$\mathrm{AB} 0127$ \\ ANTI-INFLAMMATORY AND PRO-APOPTOSIS EFFECTS OF 18BETA-GLYCYRRHETINIC ACID IN IN VIVO AND IN} VITRO MODELS OF RHEUMATOID ARTHRITIS

M. Wang ${ }^{1,2}$, R. Huang ${ }^{1}$, on behalf of Runyue Huang Group. ${ }^{1}$ Rheumatology, Guangdong Provincial Hospital of Chinese Medicine, Guangzhou, China; ${ }^{2}$ Center for Molecular Medicine, University Medical Center Utrecht, Utrecht, Netherlands

Background: Rheumatoid arthritis (RA) is one of the most common autoimmune diseases and it affects $0.5 \%-2.0 \%$ of the human population worldwide. ${ }^{1}$ Though disease modifying anti-rheumatic drugs (DMARDs) can improve the clinical condition of patients with RA, toxicities of these drugs will be accumulated with longterm use and the unwanted side effects still cannot be avoided. $18 \beta$-glycyrrhetinic acid (18 $\beta-\mathrm{GA})$, an active component of licorice, exhibits potential anti-cancer, antiinflammatory, anti-allergic, and anti-microbial activities. ${ }^{2}$ Moreover, $18 \beta-G A$ has been elucidated to attenuate hepatotoxicity or nephrotoxicity caused by chemical drugs, included methotrexate(MTX)[. ${ }^{2}$ These evidences suggested $18 \beta$-GA may become a candidate for low toxicity RA therapy as the mechanism was revealed. Objectives: The aim of this study is to investigate the underline mechanism of $18 \beta-$ GA on anti-inflammation and anti-proliferation in in vivo and in vitro models of RA.

Methods: CIA rats were treated with $18 \beta-\mathrm{GA}$, methotrexate, celecoxib and three combination therapies for 30 days. Paw swelling volume, thromboxane synthase (TxAS), proliferating cell nuclear antigenprotein (PCNA), interleukin (IL) - 1及, IL-6, and thromboxane B2 (TxB2) were detected to assess the anti-inflammation and 